\title{
Based on Load Balancing Clustering Algorithm of Wireless Sensor Network Research
}

\author{
Fengbiao Zan \\ Qinghai university for nationalities, xi'ning Qinghai Prov.810007, China \\ zanfb@163.com
}

\begin{abstract}
With the development of science and technology, information collection, transmission, processing and wireless sensor network are inseparable. In the existing wireless sensor network clustering algorithm, the typical LEACH algorithm because of its uncertainty can lead to system life is short. This paper proposes a clustering algorithm based on load balancing, the algorithm fully considering the energy consumption of cluster head nodes, and the balance of the total energy of the network. Through simulation experiments, the algorithm system life is longer, the network energy consumption efficiency is higher.
\end{abstract}

Keywords: Wireless sensor; Load balancing clustering;NS2

\section{Introduction}

In today's era of rapid development of information technology, represented by the Internet information network has brought great changes to people's life, and will continue to continue to exert its influence in every field in the future. Microelectronics, the progress of distributed computing and wireless communication technology, promoted the rapid development of low-power multifunctional sensor, in the tiny volume can integrate information acquisition, data processing and multiple functions such as wireless communication. Wireless sensor network integrated sensor technology, embedded computing technology, modern network and wireless communication technology, distributed information processing technology, etc., can collaborate through all kinds of integrated micro sensor, real-time monitoring, sensing and acquisition of various environmental or monitoring objects information, through the embedded system in information processing, and through random self-organizing wireless network with multiple hops relaying mode will perceive information transmitted to the user terminal.To truly achieve ubiquitous computing concept.

Wireless sensor network is the extension of the Internet from the virtual world to the physical world.The Internet changed the way of exchanges and communication between human beings, and the world of wireless sensor network, the information of logic and real physical fuses in together, change the way of interaction between man and nature.People can directly perceive significant world through wireless sensor network, thus greatly extend the functionality of the existing network and human cognitive ability of the world $^{[1-2]}$.

Microelectronic mechanical system refers to a collection of miniature sensors, actuators and signal processing and control circuit, interface circuit, communication and electricity comes from the integration of micro electromechanical system, the support of the micro sensor technology and enhance the capacity of the nodes of the wireless communication gives a broad application prospect for wireless sensor network, mainly in military, environmental, health, family and other business. Of course, in the special fields such as space exploration and disaster rescue, sensor network has its unique technical advantages. 


\section{The traffic monitoring}

In some big cities, traffic congestion problems become increasingly serious, traffic monitoring becomes more and more important, wireless sensor networks since the organization of low power consumption characteristics determine his play an important role in the traffic monitoring.There are now some countries use sensor network implementation for traffic monitoring, magnetic sensors, image sensors, pressure sensors retreat to more and more application in the traffic.There is also a part of colleges and universities in China to actively participate in research of wireless sensor network in traffic monitoring.

\section{Military applications}

In the field of military, sensor networks will be an integral part of the C4ISRT system.C4ISRT system goal is to use the advanced high-tech technology, for the future of modern war to design a set of command, control, communications, computing, intelligence, surveillance and reconnaissance and positioning in the integration of the battlefield command system, the military is paid great attention to the developed countries.Because sensor networks is intensive, low cost, the random distribution of nodes, self-organizing and fault-tolerant ability make it won't because some nodes in the malicious damage caused by the collapse of the whole system, it is one of the traditional sensor technology is incomparable, is this, the sensor network is very suitable for application in the harsh battlefield environment, including to monitor his own troops, equipment and supplies, monitor the conflict zone, reconnaissance enemy terrain and protection, positioning target, assessment of loss, reconnaissance and detection of nuclear, biological and chemical attack.

\section{The environmental science}

As people increasingly concerned for the environment, environmental science involved in more and more extensive. Through the traditional way to collect the raw data is a difficult job.Sensor network provided convenience for the study of the field of the randomness of data acquisition, tracking the migration of migratory birds and insects, for example, studies the influence of environmental changes on crops, monitoring ocean, atmosphere and soil composition, etc.ALERT system in several kinds of sensors to detect rainfall, river water and soil moisture, and accordingly to predict the likelihood of outbreak of flash floods.Similarly, a sensor network of forest fires are accurate and timely forecast should also be helpful.In addition, the sensor network can be applied in precision agriculture, to monitor pests of crops, soil ph and fertilization condition, etc.

\section{The smart home}

Wireless sensor network also can be used in the household system, the system through the shoes, furniture home props such as household appliances and equipment embedded semiconductor sensor, to help elderly people, and people with disabilities of family life, using wireless communication to the sensor network can efficiently transfer the necessary information to convenient take care.Smart home network system was all kinds of information related to communications equipment, household appliances and home security devices to connect family bus technology to a family of intelligent system for centralized or long-distance monitoring, control, and family affairs management, and keep the family facilities and residential environment of harmony and coordination of the system.Wireless sensor nodes distributed in need monitoring area, perform data acquisition, processing and communication, etc;Wireless enforcement agencies responsible for starting the acousto-optic alarm, camera monitoring, automatic spray, and other functions;Home wireless control center processing, forwarding information from wireless sensor nodes, and connected to the Internet for remote control.

\section{Health}

If installed in hospitalized patients with special purpose sensor nodes, such as heart rate 
and blood pressure monitoring equipment, use of sensor networks, the doctor can understand at any time by monitoring the patient's condition, to handle in time.Sensor network can be used to collect over a long period of time a person's physiological data, the data in the process of development of new drugs is very useful, and the micro sensor installed on the monitored object body won't bring too much inconvenience to people's normal life.In addition, in many aspects, such as drug management, it also has a new and unique applications.All in all, sensor network for the future of remote medical provides a more convenient and fast technology means.

\section{Related Works}

\subsection{Wireless Sensor Network}

In the wireless sensor network has three kinds of most basic entities: objectives, sensor node and observation.Goal is to apply relevant source, sensor nodes is a target information monitoring, numerous, widely distributed, but resources are limited, the node way jump no center for wireless connection between;Observation nodes as the receiver and controller, monitoring and processing network event news, distributed query request to the network or task.Under normal circumstances, and observation network in the target node can be one or more, can be static or movement;Number of sensor nodes is numerous, usually for static, which effective node is limited, and changes in the time domain and the airspace.Routing nodes is the effective information transmission to the observation of the process.

In wireless sensor network architecture, network layer routing protocol design is very important, and with the application of different routing protocols and network infrastructure is different also.Wireless sensor network layer's main goal is: finding efficient used in sensor networks routing establishment and reliable data transmission method, which makes the wireless sensor network life expectancy.Routing protocol is responsible for the data packet forward through the network from the source node to destination node, it mainly includes two aspects: function: search for optimal path between the source node and destination node, the data packet along the optimal path right forward.In wireless sensor network node energy limited and usually does not supplement, therefore routing protocols need to efficient use of energy, and sensor networks node huge number, node can only get local topology information, routing protocol to be in a local network information on the basis of choosing the right path.

Wireless sensor routing algorithm is to construct and control and sensor network topology, in general, the organization structure of wireless sensor network is divided into flat structure and hierarchical structure, the corresponding, wireless sensor routing algorithm is also divided into flat structure based routing algorithm and routing algorithm based on hierarchical structure.Wireless sensor network generally there are two kinds of structure: flat structure and hierarchical structure.Flat structure, all nodes have equal status, so can also known as peer-to-peer structure.In a hierarchical structure, the network is divided into two different types of nodes: common nodes and node, head nodes to form high level network; At the high level in the network, can divide again, again to form a higher level of network, until the highest ${ }^{[3-5]}$.

In a hierarchical structure of clustering model, the network was divided into cluster head node and ordinary node and ordinary node selection a clusters of a cluster member.Cluster head node is responsible for forwarding data between clusters.For example, when the nodes in cluster 1 to 2 nodes in the cluster B communication, node, the data is sent to A first cluster 1 cluster head; 1 cluster analysis of cluster head found in clusters of $2 \mathrm{~B}$, put forward the data to cluster 2 cluster head, cluster of cluster head of 2 after receiving the data, found that $B$ is a member of the own clusters, the data is sent to B.In order to realize the communication between the cluster head, sometimes the 
communication each other may be required for support of the gateway node, a cluster head and gateway formed a high level of the network, this is known as a virtual backbone network.

Plane of the structure of the network is simpler, without structure maintenance process.Generally exist multiple paths between source and destination, you can use multiple paths to realize the load sharing, also can choose the appropriate path for different business types.All nodes in the network are equivalent, in principle, there is no bottleneck, so more robust.The biggest disadvantage is flat structure network scale is restricted.In flat structure, each node needs to know the route to all other nodes.Because of node mobility, maintain the dynamic changes of routing information needs a lot of control message, the network size, the greater the routing maintenance overhead.Compared with the flat structure, hierarchical structure has the following advantages:

1. Improve the throughput of the system by routing information localization.Localize routing information, a hierarchical structure within the cluster nodes do not need to know the other cluster topology, a cluster topology changes not perceived by other clusters, which reduced the routing control message overhead.

2. The node localization is much more than the flat structure is simple.In the flat structure, want to know the position of a node, the need to cut the perform query operations.In a hierarchical structure, know the location of the members of the cluster of cluster head, as long as the query of the cluster head nodes can get location information.Also, a hierarchical addressing the sequence can be realized through the mobility management.According to the node and logical sequence of address for the node distribution, the relationship between clusters by the cluster head ACTS as a similar position of HLR and VLR function in cellular network management server, you can simply achieve node localization and addressing.

3. A hierarchical structure is a combination of no center and a center mode, can use two kinds of model of technology advantage. Although USES the hierarchical structure after the relative to the control center - cluster head, but the cluster head and cluster members are dynamic, node is still the network automatically.Divided into a hierarchical structure, the network is divided into relatively independent clusters, each cluster has the control center.Based on TDMA, CDMA, polling techniques can be used in the hierarchical network, routing, mobility management, network management center control techniques can also be transplanted to the wireless sensor network.

4. Because of the technology based on a center, a hierarchical structure of support for the resource management, QOS has its unique advantages.

5. Has a good scalability.It is because the hierarchical structure of performance improvement, so that with the increase of application, large-scale gradually presents the trend of the hierarchical wireless sensor network, so the focus of this thesis research is the key technology of a hierarchical self-organizing algorithm ${ }^{[6-8]}$.

\subsection{LEACH Algorithm}

LEACH first defines the concept of "round" and "cycle", round by cluster head election and stable work of two phases, and a working cycle is composed of [N/k]wheel, where $\mathrm{N}$ is the number of nodes in the network, expectations of $\mathrm{k}$ is in each round, the number of cluster head.Assume that these two parameters are preset constants, execution of the algorithm remains the same.In the election of cluster head stage, the sensor node $i$ generate a random number on the interval $[0,1] x_{\mathrm{i}}$, namely

$\boldsymbol{x}_{i}=\operatorname{Random}(\mathbf{0 , 1})$

(1)

Once the cluster head is selected, they actively to all the "neighbors" broadcast the 
news.According to the strength of the received signal, the node selection it to join the group, and inform the corresponding cluster head.Based on time division multiplexing way of cluster head time slot allocation for each member of communication. Work in the stable phase, nodes continuous monitoring data, the transmission and cluster head, cluster head to make the necessary integration.After processing, sent to the Sink node in faraway places, it is a reasonable working mode of reducing traffic. Work after a period of stable stage, the whole network into the next round, to select cluster head.After an N/k rounds, the system to complete a working cycle.

LEACH is completely distributed, nodes rely on local information only decided to own "character", is a cluster head or a regular node, and the time complexity of algorithm is $O(1)$.LEACH random cluster head election method is simple, easy to implement.It also considers the load balance problem, in a recent rounds has become a cluster head nodes will didn't have a chance to once again become a cluster head in the work cycle.It is because the LEACH has the characteristics of the above, it became the most widely used wireless sensor network clustering algorithm, and a benchmark test other clustering algorithm.

In clustering structure of the sensor network, the cluster head carries more tasks, so will consume more energy.LEACH by ensuring that in one work cycle, each node has a chance and only get one chance to become a cluster head to achieve load balancing.Although the original intention of LEACH is capitation system load, but the simulation results show that LEACH can lead to some nodes prematurely run out of battery power.Two reasons can explain this phenomenon:

1. Because of the randomness of the LEACH algorithm, some did not become A cluster head nodes were forced to direct communication with the Sink node.Although the assumption that the sensor nodes under the condition of uniform random distribution, calculated the number of the optimal value of cluster head in the system.But due to the randomness of the LEACH algorithm, so the number of cluster head is formed in each round is not always equal to the optimal value.If produce too little of the cluster head, a common node will probably not near the existence of the cluster head, so the nodes were forced to direct communication with the Sink node.Even able to generate the desired number of cluster head system, but because of the randomness of cluster head, can result in uneven distribution of cluster head, will also make some nodes and Sink without the existence of the cluster head nodes near the direct communication.

2. LEACH decision node "character" did not consider the remaining battery power of nodes, the load balancing strategy is incomplete.LEACH according to a predefined probability decision nodes at random "character".Although a node in a working cycle, only get one chance to become a cluster head, but other reasons lead to the excessive battery energy consumption, such as forced to direct communication with the Sink node, or because of the uneven distribution management too much common node, and so on.In the next work cycle of LEACH, or all of the nodes have the same opportunity to become a cluster head, the battery power is below the line of nodes will quickly run out of battery power for become a cluster head.

\section{Based on the Clustering Algorithm Design of Load Balancing}

Due to LEACH protocol cluster head election algorithm may lead to imbalance of the whole network node energy, so the algorithm in the process of cluster head election, to consider the current node energy factor, the greater the probability that the election of cluster head nodes energy bigger, so that you can to a certain extent, balance the distribution of the whole network node energy, make the network's survival time can be prolonged.The improved algorithm and LEACH the same network requirements.Or using two stage cluster formation and the stability of cluster analysis, this chapter is mainly to cluster formation stage of the method to improve the cluster head election. 
In the cluster formation stage, as mentioned above, every time is to select cluster head.The selection of cluster head also want to have a threshold $\mathrm{CH}_{\text {prob }}, \mathrm{CH}_{\text {prob }}$ here value cannot be predetermined, it has something to do with nodes of the current energy such as (2),

$$
C H_{\text {prob }}=\left(P_{\text {min }}+\left(P_{\text {max }}-P_{\text {min }}\right) \frac{E_{\text {residual }}}{E_{\text {max }}}\right)(1+\text { around })
$$

Which $\mathrm{P}_{\min }$ as the threshold value of the minimum, $\mathrm{P}_{\max }$ is the maximum threshold, $\mathrm{E}_{\text {residual }}$ for the rest of the current node energy, $\mathrm{E}_{\max }$ for the node when the initial energy. We first select an initial cluster in the $n$ nodes as a percentage of $P_{\max } \cdot P_{\max }$ is only used to restrict initial cluster head broadcasting, does not form a direct impact on the last of the cluster head, $\mathrm{CH}_{\text {prob }}$ value cannot under $\mathrm{p}_{\min }$ at the same time, the value selection and $\mathrm{E}_{\max }$ inversely proportional, set up the goal of this value is to prevent the residual $\mathrm{E}$ under the condition of relatively small, the value of the $\mathrm{CH}_{\text {prob }}$ will become smaller, so that nodes generate a random number from 0 to 1 will be difficult to smaller than $\mathrm{CH}_{\text {prob }}$, which is difficult to produce new cluster head.Round the round is the first round in each big wheels, because as the number of round, was an increase in number of nodes in the cluster head, relative to the rest of the total number of nodes to reduce, can only be guaranteed accordingly increase the value of the $\mathrm{CH}_{\text {prob }}$ in each round was elected as the number of the number of nodes in the cluster head, so on the back by a weighting factor ( $\alpha$ round +1 ), the alpha is a need to network conditions to determine an optimal value.Can be seen from the above formula, $\mathrm{CH}_{\text {prob }}$ production considering the current energy node, instead of the random number producing cluster head, so not only on the initial energy homogeneous network, and network of uneven initial energy of nodes, the algorithm will have better than LEACH algorithm of network energy balance. The whole process of the formation of clusters are shown in figure 1 below, after this is bunch of stable stage and transmission of data, the whole process is similar to LEACH.In order to prevent the communication between clusters, each cluster with different spread spectrum code for communication;And in order to prevent the interference of communications within the cluster, adopt the method of points 2 slots in each cluster to communicate, after received the data node of cluster head for data fusion, and then passed to the base station directly ${ }^{[9-12]}$.

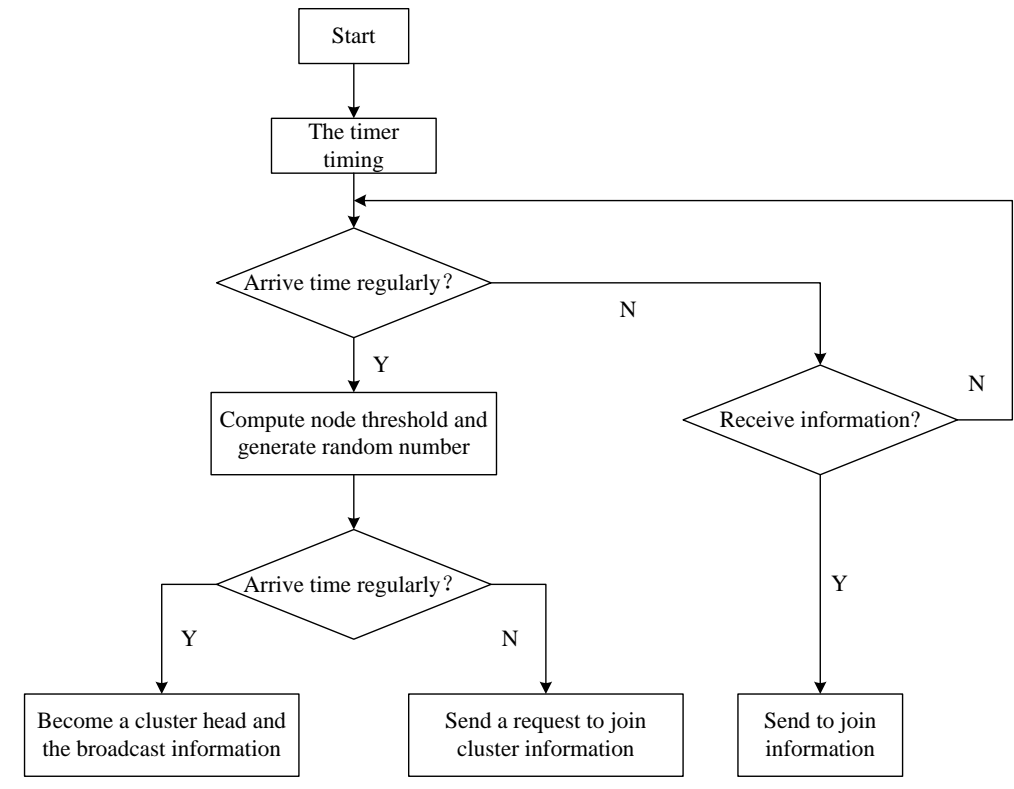

Figure 1. The Flow Chart of the Improved Cluster-Making Algorithm 


\section{The Experimental Results and Data Analysis}

In order to verify the validity of the algorithm, simulation, the algorithm using ns2 network simulation tools at $120 \mathrm{~m}$ by $120 \mathrm{~m}$ random distribution in a network of 110 nodes, each node initial with a 2.5 joules, we choose the minimum become a cluster head probability $\mathrm{P}_{\min } 0.006$. When the node number for $\mathrm{N}=110$, network boundary for $\mathrm{L}=80 \mathrm{~m}$, $\mathrm{d}=158 \mathrm{~m}$ distance from base station, the optimal number of cluster head nodes for four to six nodes, here we choose the number of initial cluster head for five, so we get $\mathrm{P}_{\max }=0.07$. Nodes of every $25 \mathrm{~s}$ time to replace round of cluster head.In the following experiment, we will verify the improved algorithm is compared with the original LEACH algorithm on the network survival time performance improvements.At the same time we also presents different alpha to network survival time ${ }^{[13-15]}$.

When using the improved algorithm, the parameter $\mathrm{P}_{\min }=0.006, \mathrm{P}_{\max }=0.07, \alpha=0.15$, the number of nodes in the network of active distribution are shown in figure 2 below:



Figure 2. The Relation between the Number of Active Node and Time

Can be seen from the figure 2, the original LEACH algorithm in time of death is probably the first node of the $150 \mathrm{~s}$, the first node of the improved algorithms of time of death is around in the $300 \mathrm{~s}$, the improved algorithm on the network survival time have greatly improved, and the curve is relatively steep decline, this is also the result of the network energy balance, this is because many small energy of nodes in a certain time network run out at the same time, the phenomenon.

In sensor networks, the nodes to calculate the energy storage is small, mainly from energy consumption in data transmission, the energy consumption of hierarchical routing algorithm can be divided into two aspects: one is the stage of clusters of energy consumption, is a bunch of stable phase data transmission of energy consumption.In the stage of clusters, cluster head needs to send data receiving a twice, the energy dissipations of the cluster head is roughly can be expressed in the following formula: $E_{e}=2 k\left(E_{t x}+E_{a} d^{2}\right)+k E_{r x}$

Which the number $\mathrm{k}$ for the members of the cluster head, Etx for sending node energy, Erx for receiving node energy, Ea for amplifier consume of energy, the distance $d$ for the node to send 


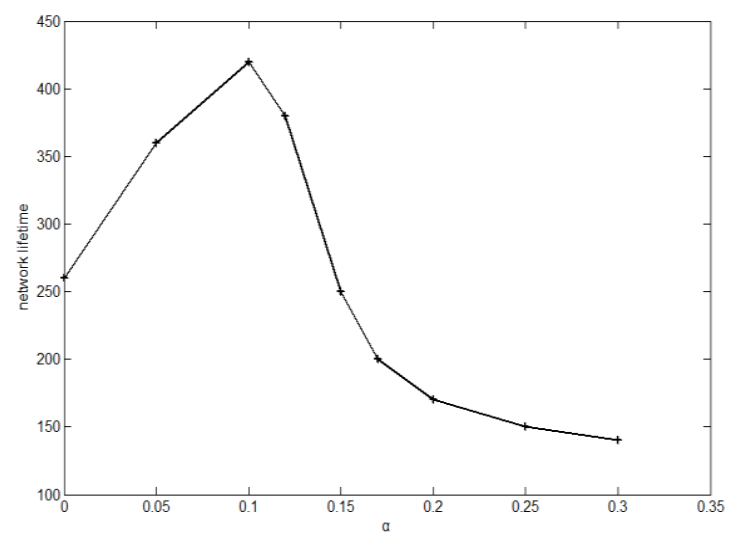

Figure 3. The Relation between the Life Time of Network and $\alpha$

Can be seen from figure 3 , when $\alpha$ is small, the relatively small number of cluster head in each round, so the members of each cluster is more, the k value is bigger, cluster head node energy consumption is relatively large, relatively small network survival time.In data transmission phase, because the distance between cluster head and base station is bigger, the cluster head and base station communication energy consumption is larger, when $\alpha$ is larger, the network cluster head is much, can also lead to the network energy imbalance, influence the survival time of the network.

\section{Conclusion}

Based on the analysis of LEACH agreement, on the basis of cluster head selection algorithm shortcoming, this paper proposes a new cluster head selection algorithm, this algorithm considering the current node when choosing threshold energy, so that can be balanced in the communication of the whole network energy.The simulation results show that the algorithm is compared with the original LEACH algorithm in improving network has obvious effect on the survival time.At the same time, this algorithm is simpler, can be easily transplanted to wireless sensor network hardware platform, has been applied in practice.

\section{Acknowledgements}

This work is supported by the Chunhui planning project of Ministry of Education of China under Grant Z2010073.

\section{References}

[1] Oreku G S, Pazynyuk T. Security in Wireless Sensor Networks[M]. Springer International Publishing, 2016.

[2] Li P, Sun L, Fu X, et al. Security in Wireless Sensor Networks[M]// Wireless Network Security. Springer Berlin Heidelberg, 2013:179-227.

[3] Surhone L M, Tennoe M T, Henssonow S F, et al. Wireless Sensor Network[M]. Betascript Publishing, 2013.

[4] Li J, Zeng P, Zong X, et al. Communication and control co-design for wireless sensor networked control systems[C]// Intelligent Control and Automation (WCICA), 2014 11th World Congress on. IEEE, 2015:156-161.

[5] Branch J W, Giannella C, Szymanski B, et al. In-network outlier detection in wireless sensor networks[J]. Knowledge \& Information Systems, 2013, 34(1):23-54.

[6] Amara S O. Securing Wireless Sensor Networks: A Survey[J]. Edpacs the Edp Audit Control \& Security Newsletter, 2013, 47(2):6-29.

[7] Baccour N, Koub\&\#, Anis A, et al. Radio link quality estimation in wireless sensor networks: A survey[J]. Acm Transactions on Sensor Networks, 2012, 8(4):688-688.

[8] Srisooksai T, Keamarungsi K, Lamsrichan P, et al. Practical data compression in wireless sensor 
networks: A survey[J]. Journal of Network \& Computer Applications, 2012, 35(1):37-59.

[9] Pang B M, Shi H S, Li Y X. An Energy-Efficient MAC Protocol for Wireless Sensor Network[J]. Lecture Notes in Electrical Engineering, 2012, 4:V4-619 - V4-623.

[10] Zhang H, Li L, Yan X F, et al. A Load-balancing Clustering Algorithm of WSN for Data Gathering[C]// Artificial Intelligence, Management Science and Electronic Commerce (AIMSEC), 2011 2nd International Conference on. IEEE, 2011:915-918.

[11] Li-Juan D, Hong-Jun J. Load Balancing Clustering Algorithm in Mobile Grid[C]// 2013 Third International Conference on Intelligent System Design and Engineering Applications. IEEE, 2010:915-918.

[12] Newton C, Yevmenkin M, Fullagar D, et al. LOAD-BALANCING CLUSTER: WO, US20130173806[P]. 2013.

[13] YANG Shun tao. The Design about the High-performance Load Balancing Cluster System based on LVS[J]. Journal of Guangxi University for Nationalities, 2012.

[14] Liu N, WENG Chu liang, LI Ming lu. Performance benchmark methodology for load balancing cluster[J]. Computer Engineering \& Design, 2011, 32(10):3407-3398.

[15] Gourley D. An apache load balancing cluster[J]. Sys Admin, 2001, 10(1):8-16.

\section{Author}

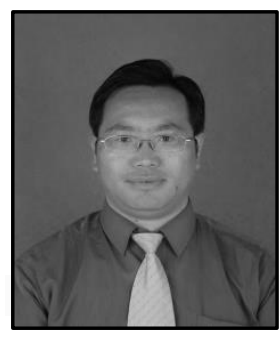

Fengbiao ZAN receives the M.S. degree in college of computer science and engineering at University of Electronic Science and Technology of China. He is a professor with school of computer, Qinghai University for Nationalities. . His research interests include wireless sensor network, communication and computer network. 
International Journal of Grid and Distributed Computing Vol.9, No.5 (2016) 